\title{
Population Level Assessments of Immunization- When More Complex is Better
}

\author{
STEPHEN Soster \\ National Polio Surveillance Project, World Health Organization, India.soslerst@npsuindia.org
}

I $\mathrm{t}$ is estimated that more than 10 million children in India do not receive BCG, 3 doses of OPV and DTP, and measles vaccine during their first year of life and more than 3 million of these do not receive any immunizations[1]. The complexity and diversity of India means that the distribution of unvaccinated and partially vaccinated children is not equal either between or within states. How immunization performance gaps are bridged is of interest to all stakeholders - Union and State governments and immunization partners. Bihar, India's second more populous state historically competed for the inauspicious distinction of having the lowest immunization coverage rates in the country. To be certain, times have changed and Bihar's progress to improve vaccination coverage over the past several years has been considerable. For these reasons, the assessment by Goel and colleagues of Bihar's Muskaan Ek Abhiyan campaign [2] is timely and welcome.

The article presents the experience of Bihar's Muskaan Ek Abhiyan and compares survey data from other Empowered Action Group (EAG) states to assess immunization coverage improvement. The conclusions of the authors, namely that there was marked improvement in immunization coverage and there are replicable lessons to learn from Bihar's experience, are incontrovertible. Coinciding with a change of government and the design and implementation of Muskaan in 2005 , vaccination coverage rates have climbed steadily, demonstrating that improving India's Universal Immunization Program (UIP) is possible in the most intractable areas.

Unfortunately, this assessment falls short of what is needed, namely a comprehensive in-depth analysis of the effectiveness of Muskaan. Instead, the report relies on a normative description of its interventions and state-wide comparison of survey assessed vaccination coverage to make its point. The authors correctly point out that it "is difficult to extract the effect of individual interventions of Muskaan." Such an assessment is difficult; but it is not impossible. It is precisely this disaggregation of individual components of Muskaan that is needed to provide useful insight to the relative merits of interventions and the mechanisms that affect vaccination coverage [3,4]. Rather than the fault of the authors, this situation illustrates the need to advocate for the use of appropriate methodologies when evaluating large scale programs, as well as - and above all - the need for valid and reliable data from multiple sources in order to do so.

India's Universal Immunization Program (UIP) targets an estimated annual live-birth cohort of 26 million children and faces multiple challenges that include high drop outs rates, a dearth of trained human resources at all levels, periodic vaccine stock-outs, infrequent supervision, unsafe waste disposal practices of injection material and inconsistent communication promoting immunization [5]. In addition, India's UIP also suffers from a lack of reliable, well organized program data to permit robust monitoring and evaluation to guide UIP strategic planning and policy formulation. There is a desperate need to improve the accuracy, completeness, timeliness and feedback of UIP-related data at all levels. The state-of-the-art Health Management Information System (HMIS), designed to collect service statistics and program related information for numerous programs at the district level, generates data that often bears little resemblance to reality and is of little use to program managers. The ultimate impact of immunization efforts is reduced morbidity and mortality due to vaccine preventable diseases. Yet for the exception of acute flaccid paralysis surveillance for poliomyelitis, effective and appropriate national surveillance does not exist to provide accurate burden of disease information and trends for the target diseases of current UIP antigens. Moreover, plans have not materialized to establish sentinel surveillance sites to capture disease-specific data related to new and important vaccines such as Haemophilus influenzae type $\mathrm{b}$, pneumococcal and rotavirus vaccines. Monitoring of immunization session-sites and community coverage, where it's been implemented, provides real-time assessment of the quality and reach of immunization 
services. Bihar has implemented vaccination session-site and community monitoring and together with partners provided feedback on 39,051 immunizations sessions monitored and the vaccination status of 310,843 children 0-23 months of age [6]. In a more robust assessment of Muskaan, this information would be crucial to better understand the intermediate pathways associated with the uptake of immunization services. Unfortunately, Bihar is just one of a handful of states that has implemented this system of monitoring. Lastly, strategies and intensity of activities required to increase coverage from 30 to 50 percent will not be the same when attempting to raise coverage to 90 percent and above. For this reason, it is also necessary to link budget and expenditure data to aid decisions related to the costeffectiveness of different interventions.

Over the last several years, Bihar has more than doubled its proportion of fully immunized children while aggressively pursuing polio eradication at a level of intensity rivaled only in Uttar Pradesh. Untangling the mixed effects in National Rural Health Mission (NRHM), Muskaan, polio eradication efforts and the role of Government strength and ownership is a complex endeavor that requires robust prospective program evaluation informed by multiple data sources. As well known evaluators have aptly stated, "Evaluations of complex population-level interventions are likely to have complex answers, but are necessary to improve future programs."'[7]

Funding: None; Competing interests: The views expressed in this paper are those of the author alone and should not be attributed to the World Health Organization.

\section{REFERENCES}

1. UNICEF Coverage Evaluation Survey. All India Report. New Delhi: UNICEF; 2009.

2. Goel S, Dogra V, Gupta SK, Lakshmi PVM, Varkey S, Pradhan N, et al. Effectiveness of Muskaan Ek Abhiyan (The Smile Campaign) for Strengthening Routine Immunization in Bihar, India. Indian Pediatr 2012; 49: 103-8.

3. Ng M, Gakidou E, Levin-Rector A, Khera A, Murray CJ, Dandona L. Assessment of population-level effect of Avahan, an HIV-prevention initiative in India. Lancet.2011;378:1643-52.

4. Victora CG, Black RE, Boerma T, Bryce J. Measuring impact in the Millennium Development Goal era and beyond: a new approach to large-scale effectiveness evaluations. The Lancet. 2011;377:85-95.

5. Sagar KS. Improving UIP Coverage in India, concept note. New Delhi: Maternal and Child Health Integrated Program (MCHIP); 2011.

6. National Polio Surveillance Project. Routine Immunization Monitoring database. New Delhi: NPSP, WHO: 2010-2011.

7. Boerma T, d Zoysa I. Beyond accountability: learning from large-scale evaluations. The Lancet. 2011;378:1610-1.

\title{
Low Serum Magnesium and Obesity - Causal Role or Diet Biomarker?
}

\author{
Anura V Kurpad And Isabelle Aeberli \\ Division of Nutrition, St John's Research Institute, Bangalore 560034, India. \\ a.kurpad@sjri.res.in
}

$\mathrm{T}$ he association between a low magnesium $(\mathrm{Mg})$ status, and insulin resistance and the metabolic syndrome has been shown repeatedly in adults [1], and higher Mg intakes have been shown to have a protective role [2]. This leads to the tantalizing conclusion of a mechanistic involvement of $\mathrm{Mg}$ in weight gain and insulin resistance through its critical role as a cofactor in several enzymes in carbohydrate metabolism [1]. However, the $\mathrm{K}_{\mathrm{m}}$ of $\mathrm{Mg}$ for these enzymes is close to its intracellular concentration, which does not decline easily on $\mathrm{Mg}$ deficient diets [3]. The effect is also subtle: the adjusted risk for diabetes with quite low $(<1.7 \mathrm{mg} / \mathrm{dL})$ serum $\mathrm{Mg}$ levels have been shown to increase modestly by about 1.5 fold [4]. Furthermore, reverse causality is also possible: diabetes is known to increase renal $\mathrm{Mg}$ excretion, and insulin resistance decreases Mg uptake. Therefore, the theoretical framework that links low Mg status to obesity and insulin resistance is not simple.

The relation between obesity and $\mathrm{Mg}$ is also not clear. Several studies have assessed the association between body mass index and $\mathrm{Mg}$ intake or status in adults, but the results are still controversial [2,5]. This may be because the associations of low serum $\mathrm{Mg}$ with obesity related outcomes are subtle and subject to type 2 errors. In this issue of Indian Pediatrics, Jose, et al. [6] have found that serum $\mathrm{Mg}$ was lower in obese Indian children, but this counter-intuitively occurred with apparently higher $\mathrm{Mg}$ intakes. The authors are careful not to imply any mechanistic linkage either way, but speculate that the 
aggravation of the insulin resistant state with low serum $\mathrm{Mg}$ could start early in childhood.

Measuring Mg status accurately is challenging, since serum $\mathrm{Mg}$ is only about $1 \%$ of the total body $\mathrm{Mg}$, and most probably reflects its renal handling rather than its dietary intake. A very low $\mathrm{Mg} \operatorname{diet}(<10 \%)$ in a human subject in 'excellent health' led to a drop by about $0.4 \mathrm{mg} / \mathrm{dL}$ in serum levels, along with negative balances, but no drop in intracellular levels [3]. On the other hand, intracellular $\mathrm{Mg}$ depletion has been found with normal serum $\mathrm{Mg}$ concentrations [1]. The point is that $\mathrm{Mg}$ intake cannot easily be related to serum level or status. Pre-analytical factors, including the effect of prior exercise on serum $\mathrm{Mg}$, are also important.

Nevertheless, given the observation that the obese children had a higher energy adjusted Mg intake [6], the low serum $\mathrm{Mg}$ levels are likely to be an 'effect' rather than a cause. The authors' opinion is that the observed differences could have been due to decreased $\mathrm{Mg}$ absorption or increased excretion. Both these mechanisms are plausible. The questions relate to how and why. For example, one could enquire whether the obese children had a higher calcium $(\mathrm{Ca})$ intake, since this is known to interfere with $\mathrm{Mg}$ absorption. Indeed, dairy products are high in $\mathrm{Ca}$ and low in $\mathrm{Mg}$ content. The intake of carbonated soft drinks, with higher intakes of phosphorus could also interfere with absorption while caffeine can increase renal $\mathrm{Mg}$ excretion [7]. A vegetarian and unprocessed food-based diet, such as with whole grains, nuts, and green leafy vegetables, is high in $\mathrm{Mg}$, which is lost during processing. Therefore, in studies that investigate associations between serum $\mathrm{Mg}$ and other outcomes, it is critical to have a close inspection of the dietary environment.
Observational studies such as those by Jose, et al. [6] are important in a transitioning society with changing processed food intake; however, longitudinal studies with detailed food intake assessment are required to assign causality or to assess the potential interaction with insulin resistance. Until then, the role of $\mathrm{Mg}$ will remain enigmatic, the need for supplements unclear, and serum $\mathrm{Mg}$ may simply have to continue to be considered as a biomarker for a particular type of diet.

Competing interests: Dr Kurpad is a member of the Kraft Health and Wellness Global Advisory Board; Funding: None.

\section{REFERENCES}

1. Barbagallo M, Dominguez LJ. Magnesium metabolism in type 2 diabetes mellitus, metabolic syndrome and insulin resistance. Arch Biochem Biophys. 2007;458:40-7.

2. He K, Liu K, Daviglus ML, Morris SJ, Loria CM, Van Horn $\mathrm{L}$, et al. Magnesium intake and incidence of metabolic syndrome among young adults. Circulation. 2006;113:1675-82.

3. Dunn MJ, Walser M. Magnesium depletion in normal man. Metabolism. 1966;15:884-95.

4. Kao WH, Folsom AR, Nieto FJ, Mo JP, Watson RL, Brancati FL. Serum and dietary magnesium and the risk for type 2 diabetes mellitus: The Atherosclerosis Risk in Communities Study. Arch Intern Med. 1999;159:2151-9.

5. Song Y, Sesso HD, Manson JE, Cook NR, Buring JE, Liu S. Dietary magnesium intake and risk of incident hypertension among middle-aged and older US women in a 10-year follow-up study. Am J Cardiol. 2006;98:1616-21.

6. Jose B, Jain V, Vikram NK, Agarwala A, Saini S. Serum magnesium in overweight children. Indian Pediatr. 2012, 49:109-12.

7. Swaminathan R. Magnesium metabolism and its disorders. Clin Biochem Rev. 2003;24:47-66.

\title{
Environmental Exposures and Childhood Cancer
}

\author{
GAURI KAPOOR \\ Pediatric Hematology Oncology, Rajiv Gandhi Cancer Institute \& Research Center, Sec -V, Rohini, Delhi-110085. \\ gauri_kapoor2000@yahoo.com
}

The link between environmental agents and childhood cancer is not a new concept. Environmental causes of childhood cancer have long been suspected by many scientists but have been difficult to pin down, partly because cancer in children is rare and because it is difficult to identify past exposure levels in children, particularly during potentially important periods such as pregnancy, in-utero, or even prior to conception. Hence, many of the environmental agents hypothesized for childhood leukemia remain speculative [1-3].

In this issue of Indian Pediatrics, Rau et al. [4] investigated the presence of endosulfan in the bone marrow of children with hematological malignancy residing in areas sprayed with the pesticide (in South India). This is a case-control study in which the authors 
report its presence in the bone marrow of $7 / 34$ children residing in these areas including 6 who had leukemia.

The authors themselves point out that this study does not in any way prove that endosulfan is a cause of leukemia. As of date, there are no available epidemiological studies linking endosulfan exposure specifically to cancer in humans. It is important to understand that an association between an exposure and cancer does not necessarily mean that the exposure causes cancer. Most importantly, other possible explanations of the observed association must be ruled out. The consistency of an association is to be considered, and the association must be temporally correct meaning that we must be sure that the exposure actually preceded the development of the disease, which in this case would include events taking place before birth, during conception, embryogenesis and early postnatal life. Another important aspect in such a study is the sample size and whether it is truly representative of the population. As acknowledged by the authors, the small sample size, other compounding factors like genetic susceptibility, exposure to other carcinogens and their effects, are the limitations of this study.

Endosulfan, an off patent organochlorine insecticide and acaricide was developed in the early 1950s and has been used globally in agriculture to control insect pests. Although industrialized nations have restricted or banned many organochlorine pesticides, some of these chemicals (like endosulfans) are still used, on the assumption that they pose little threat to the environment, wildlife, or human health.

It has long been recognized that leukemia is a heterogenous disease with a multifactorial and multistep pathogenesis with a fetal origin being postulated by some $[3,5]$. Epidemiological evidence suggests that ionizing radiation, certain chemicals (such as benzene), viruses (human T-cell leukemia/lymphoma virus type I, Epstein-Barr virus), and bacteria (Helicobacter pylori) may play a part in the development of some subtypes of leukemia and lymphoma in adults and children.

Finding causes of any disease is usually a long, slow process. No one study is likely to prove that a particular exposure definitely causes a particular cancer. However, each well designed and well executed study with adequate sample size will bring us closer to understanding the causes of these cancers within populations of children.

Competing interests: None stated; Funding: Nil.

\section{REFERENCES}

1. Pearce MS, Hammal DM, Dorak MT, McNally RJ, Parker L. Paternal occupational exposure to pesticides or herbicides as risk factors for cancer in children and young adults: a case-control study from the North of England. Arch Environ Occup Health. 2006;61:138-44.

2. UK Childhood Cancer Study Investigators. Childhood cancer and residential proximity to power lines. $\mathrm{Br} \mathrm{J}$ Cancer. 2000;83:1573-80.

3. Toren A, Rechavi G, Ramot B. Pediatric cancer: Environmental and genetic aspects. Ped Hematol Oncol. 1996;13:319-31.

4. Rau A, Coutinho A, Avabratha KS, Rau AR, Warrier RP. Pesticide (endosulfan) levels in the bone marrow of children with hematological malignancies. Indian Pediatr. 2012;49:113-7.

5. Wiemels JL, Cazzaniga G, Daniotti M, Eden OB, Addison GM, Masera G, et al. Prenatal origin of acute lymphoblastic leukaemia in children. Lancet. 1999; 354:1499-1503.

6. Greaves M. Molecular genetics, natural history and the demise of childhood leukaemia. Eur J Cancer. 1999; $35: 173-85$. 